\title{
Selection of Heating System at the Reconstruction of a Family House
}

\author{
Peter Petrilák, Ladislav Böszörményi \\ Technical University of Košice \\ Civil Engineering Faculty, Institute of Architectural Engineering \\ e-mail: peter.petrilak@tuke.sk, ladislav.boszormenyi@tuke.sk
}

\begin{abstract}
The work deals with the reconstruction of a family house on the part of energy for heating need. The attention was devoted to comparise individual alternatives, which are possible to realise in assessed house. In the first part of the work, the attention is devoted to describing object and to heat loses values before and after the measurements. Then alternatives of heating their annual costs for heat production and for amount of fuel, invested costs and the portion of invested and overall are particularly characterized. In the part: The economical evaluation of the comparised alternatives, simple return of investments outputs, discount time of return, net actual value and yield rate are. These values give information about the expedience of applications of individual alternatives by economical aspect.
\end{abstract}

Key words: heating systems, condensing gas boiler, heat pump, biomass

\section{Introduction}

Currently dominated by the amount of energy speculation by investors how to ensure or improve the heating system in their new or existing family house. This is primarily about saving energy, respectively reduction of the amount energy for heating. The gas crisis is the willingness of investors to be the least dependent on the fossil fuel in case of strangulated valve for a longer period, which would result a shortage of gas for households. In this paper, attention is paid to choosing the most appropriate heating system for the existing family house of the five considered alternatives.

\section{Description of the assessed family house}

The presented building, shown in figure 1, was built in the 80's of the last century. No structural modifications, implying the current thermal characteristics of packaging structures, 
were in the house. In this state, the facility, the factors set out in the heat transfers $\left[\mathrm{W} \cdot \mathrm{m}^{-2} \cdot \mathrm{K}^{-}\right.$ ${ }^{1}$ ], which were used in the building heat loss calculation. The construction modifications shall be proportionately reduced and the heat loss of the object, shown in Table 1. According to the lowest possible investment cost will be the thickness of the contact thermal insulation system way intended to be used already existing heaters in the house even when the temperature gradient reduced from the existing $90 / 70{ }^{\circ} \mathrm{C}$ to $55 / 45^{\circ} \mathrm{C}$. This temperature gradient is suitable for the better use of condensation in the gas condensing boiler or heat pump. The system of heat pump will be water/water, which works with the highest efficiency among all types of heat pumps. Table 1 shows the performance of radiators in these temperature gradients. There are used sectional cast iron radiators.

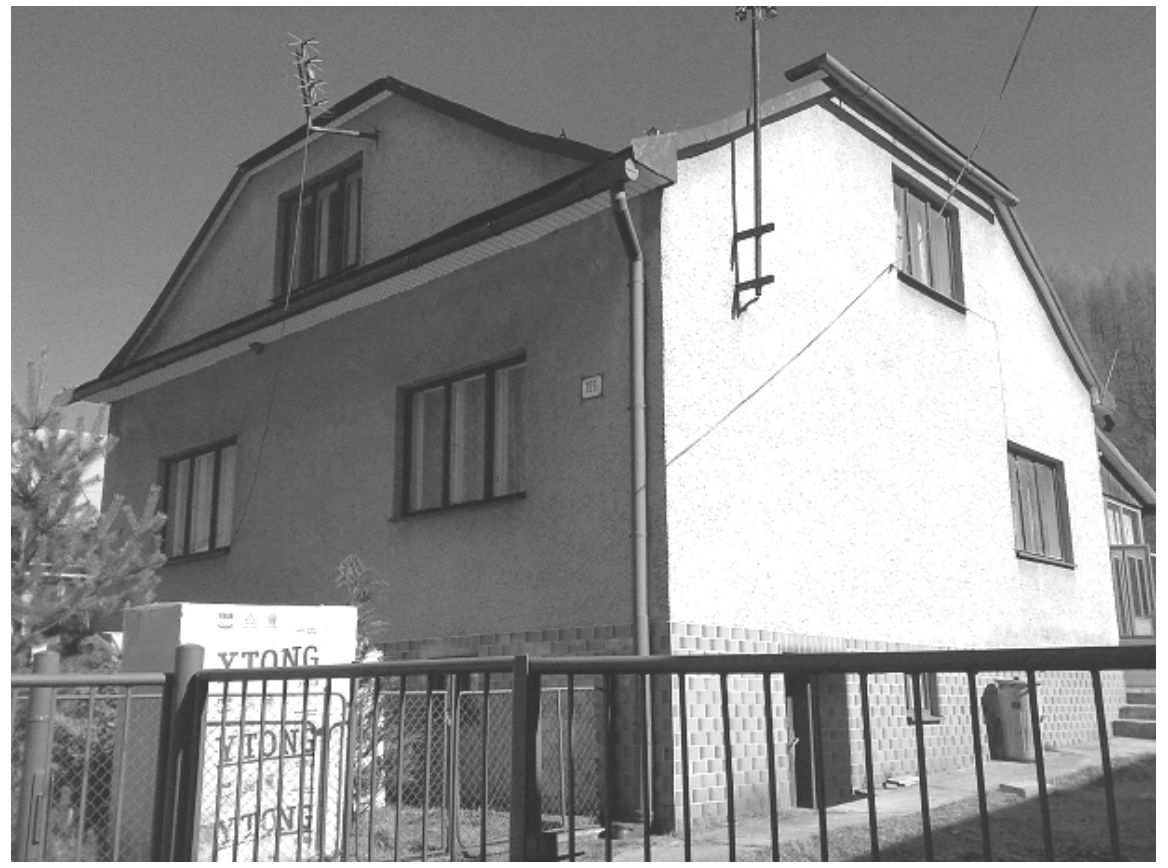

Figure 1: Assessed family house

Table 1: Heat loss for the current status and the construction modifications

\begin{tabular}{|c|c|c|c|c|}
\hline & $\begin{array}{c}\text { Present: } \\
\text { Heat loss } \\
\text { [W] }\end{array}$ & $\begin{array}{c}\text { Performance } \\
\text { radiators for the } \\
\text { temperature } \\
\text { gradient }\end{array}$ & $\begin{array}{c}\text { After warm-ups } \\
\text { and exchange: } \\
\text { Heat loss [W] }\end{array}$ & $\begin{array}{c}\text { Performance } \\
\text { radiators for the } \\
\text { temperature } \\
\text { gradient }\end{array}$ \\
\cline { 2 - 5 } & Qc & $90 / 70^{\circ} \mathrm{C}[\mathrm{W}]$ & Qc & $55 / 45^{\circ} \mathrm{C}[\mathrm{W}]$ \\
\hline 1-st floor & 11707 & 12181 & 6476 & 6907 \\
\hline 2-nd floor & 6447 & 6792 & 2970 & 3719 \\
\hline overall & $\mathbf{1 8 1 5 4}$ & $\mathbf{1 8 9 7 3}$ & $\mathbf{9 4 4 6}$ & $\mathbf{1 0 6 2 6}$ \\
\hline
\end{tabular}

Because of the insulation system is used only at the facade of the building, reducing the rate of heat loss is not constant for each room. In the more the surface masonry room will have, the lower the rate of heating required will be after isolation. Therefore, in some existing 
rooms the radiators remain in situ; in the other rooms will be confused. By using existing radiators investor saves $€ 860.4$ and not only that: as cast iron material is characterized by high thermal inertia, so they can transmit heat, although it is no longer a source of heat put into dipped regime. The new two radiators will be purchased. The price is $€$ 311.8. Two radiators remain unused because of they have low performance at a reduced temperature gradient $55 / 45^{\circ} \mathrm{C}$.

\section{Alternatives solutions of heating}

Table 2: Description of the alternatives heating

\begin{tabular}{|l|c|c|c|c|c|c|}
\hline & $\begin{array}{c}\text { Initial } \\
\text { state }\end{array}$ & $\begin{array}{c}\text { Altern. } \\
\mathbf{1}\end{array}$ & $\begin{array}{c}\text { Altern. } \\
\mathbf{2}\end{array}$ & $\begin{array}{c}\text { Altern. } \\
\mathbf{3}\end{array}$ & $\begin{array}{c}\text { Altern. } \\
\mathbf{4}\end{array}$ & $\begin{array}{c}\text { Altern. } \\
\mathbf{5}\end{array}$ \\
\hline The original boiler & $\mathbf{X}$ & - & - & - & - & - \\
\hline New condensing gas boiler & - & $\mathrm{X}$ & $\mathrm{X}$ & - & - & - \\
\hline New boiler for wood pellets & - & - & - & - & $\mathrm{X}$ & $\mathrm{X}$ \\
\hline Heat pump water-water & - & - & - & $\mathrm{X}$ & - & - \\
\hline Initial distribution & $\mathrm{X}$ & $\mathrm{X}$ & $\mathrm{X}$ & $\mathrm{X}$ & $\mathrm{X}$ & $\mathrm{X}$ \\
\hline Old radiators & $\mathrm{X}$ & $\mathrm{X}$ & - & - & $\mathrm{X}$ & - \\
\hline Buying + substitution radiator & - & - & $\mathrm{X}$ & $\mathrm{X}$ & - & $\mathrm{X}$ \\
\hline Hydraulic regulation & - & $\mathrm{X}$ & $\mathrm{X}$ & $\mathrm{X}$ & $\mathrm{X}$ & $\mathrm{X}$ \\
\hline Temp. gradient $90 / 70^{\circ} \mathrm{C}$ & $\mathrm{X}$ & $\mathrm{X}$ & - & - & $\mathrm{X}$ & - \\
\hline Temp. gradient $55 / 45^{\circ} \mathrm{C}$ & - & - & $\mathrm{X}$ & $\mathrm{X}$ & - & $\mathrm{X}$ \\
\hline Building cladding & - & - & $\mathrm{X}$ & $\mathrm{X}$ & - & $\mathrm{X}$ \\
\hline Replacement windows and doors & - & - & $\mathrm{X}$ & $\mathrm{X}$ & - & $\mathrm{X}$ \\
\hline
\end{tabular}

In the house are heated 11 rooms, located at 1st and 2nd floor. Basement is not heated. Therefore, in the basements rooms considering temperature $5{ }^{\circ} \mathrm{C}$. In the boiler room, located at 1st floor is located the original boiler, which is necessary to replace what has been incentive to the development of various alternative options for heating. Various options are found in Table 2.

\section{Operating costs and total capital}

\subsection{Annual costs for heat and fuel}

Table 3: The annual need for heat and fuel

\begin{tabular}{|l|c|c|c|c|c|c|}
\hline & $\begin{array}{c}\text { Initial } \\
\text { state }\end{array}$ & $\begin{array}{c}\text { Altern. } \\
\mathbf{1}\end{array}$ & $\begin{array}{c}\text { Altern. } \\
\mathbf{2}\end{array}$ & $\begin{array}{c}\text { Altern. } \\
\mathbf{3}\end{array}$ & $\begin{array}{c}\text { Altern. } \\
\mathbf{4}\end{array}$ & $\begin{array}{c}\text { Altern. } \\
\mathbf{5}\end{array}$ \\
\hline The annual need for heat $-\mathrm{Q}_{\mathrm{or}}[\mathrm{MJ}]$ & 159740 & 159740 & 70553 & 70553 & 159740 & 70553 \\
\hline Annual need for gas $-\mathrm{U}_{\mathrm{d}}\left[\mathrm{Nm}^{3}\right]$ & 6275 & 5298 & 2340 & 0 & 0 & 0 \\
\hline Annual need for wood pellets $-\mathrm{U}_{\mathrm{d}}[\mathrm{kg}]$ & 0 & 0 & 0 & 0 & 10692 & 4722 \\
\hline
\end{tabular}


Annual operating costs are in Table 4. For the current state as well as the alternatives has been assigned the type of tariff and the energy consumption. Regarding the electricity consumption, a family house is currently engaged in two-way rate AKU Maxi M, which means that the energy import is divided into a high tariff and low tariff, which lasts 8 hours a day - in the night-time. The alternative 3 uses the heat pump. In this case, the family house is moved into the two-way rate for the sampling locations with a heat pump, where the rate of low tariff is provided daily for a total duration of at least 22 hours. The distribution system operator shall determine the period of 2 hours of high tariff. In all cases, the hot water is provided by electric storage device that is switched at low tariff.

For all alternatives are calculated values of annual heating needs and the annual fuel needs. Calculated values are listed in Table 3.

Table 4: Conditions and charges for collection of gas, electricity respectively wood pellets, and total operating costs

\begin{tabular}{|c|c|c|c|c|c|c|}
\hline & $\begin{array}{c}\text { Initial } \\
\text { state }\end{array}$ & $\begin{array}{c}\text { Altern. } \\
1\end{array}$ & $\begin{array}{l}\text { Altern. } \\
2\end{array}$ & $\begin{array}{c}\text { Altern. } \\
3\end{array}$ & $\begin{array}{c}\text { Altern. } \\
4\end{array}$ & $\begin{array}{l}\text { Altern. } \\
5\end{array}$ \\
\hline Type of customer gas & Home & Home & Home & - & - & - \\
\hline $\begin{array}{l}\text { Estimated annual consumption of } \\
\text { gas }\left[\mathrm{m}^{3}\right]\end{array}$ & 6275 & 5298 & 2340 & - & - & - \\
\hline Recommended type of tariff (gas) & D3 & D3 & D3 & - & - & - \\
\hline $\begin{array}{l}\text { The amount of energy in the gas } \\
\text { [kWh] }\end{array}$ & 62962 & 53151 & 23474 & - & - & - \\
\hline $\begin{array}{l}\text { Estimated pre-payment for gas } \\
\text { (year) [€] }\end{array}$ & 2782 & 2363 & 1095 & - & - & - \\
\hline $\begin{array}{l}\text { Recommended type of } \\
\text { involvement (electricity) }\end{array}$ & $\begin{array}{c}\text { AKU } \\
\text { Maxi M }\end{array}$ & $\begin{array}{c}\text { AKU } \\
\text { Maxi M }\end{array}$ & $\begin{array}{c}\text { AKU } \\
\text { Maxi M }\end{array}$ & $\begin{array}{c}\text { EKO } \\
\text { Dom M }\end{array}$ & $\begin{array}{c}\text { AKU } \\
\text { Maxi M }\end{array}$ & $\begin{array}{c}\text { AKU } \\
\text { Maxi M }\end{array}$ \\
\hline $\begin{array}{l}\text { The annual fee for a sampling } \\
\text { point [€/ year] }\end{array}$ & 102,37 & 102,37 & 102,37 & 47,00 & 102,37 & 102,37 \\
\hline $\begin{array}{l}\text { Collection of electricity annually } \\
\text { HP LT [kWh / year] }\end{array}$ & - & - & - & 5400 & - & - \\
\hline $\begin{array}{l}\text { Collection of other sources of } \\
\text { electricity in the building of LT } \\
\text { [kWh / year] }\end{array}$ & 4057,03 & 4057,03 & 4057,03 & 3802,03 & 4057,03 & 4057,03 \\
\hline $\begin{array}{l}\text { Collection of other sources of } \\
\text { electricity in the building of HT } \\
\text { [kWh / year] }\end{array}$ & 5537,35 & 5537,35 & 5537,35 & 5027,35 & 5537,35 & 5537,35 \\
\hline Annual payment for electricity [€] & 1015,2 & 1015,2 & 1015,2 & 1488,4 & 1015,2 & 1015,2 \\
\hline $\begin{array}{l}\text { Annual payment for wood pellets } \\
\text { (including delivery) }[€]\end{array}$ & - & - & - & - & 1390,0 & 613,9 \\
\hline Total operating expenses [€] & 3797,2 & 3378,2 & 2110,2 & 1488,4 & 2507,6 & 1731,4 \\
\hline
\end{tabular}

\subsection{Investment costs}

The calculation of return may significantly affect the investment costs, which the illusion about calculation of the operating costs can adjust right mirror. Table 5 shows the investment costs, excluding VAT, where is included the fee for the work. Next to the house is located existing well, that were previously the sole source of drinking water. The village was 
established by a public water supply, allowing the family house to connect to the source of drinking water. In alternative 3 will be well utilized as sourced or absorbed for heat pump. The method uses a well determined to build the second exploration well.

\subsection{The share of annual investment and total cost}

Comparison of annual investment and total cost of all alternatives will be considered with the fact that the economic life of the heating system is 20 years. Usually this period is extended to a longer period.

Investment costs and annual total annual costs for all alternatives are listed in Table 5. Figure 2 shows a bar graph with the annual investment and operating costs for all alternatives.

Table 5: The total investment, annual investment and total annual cost

\begin{tabular}{|l|c|c|c|c|c|c|}
\hline & $\begin{array}{c}\text { Initial } \\
\text { state }\end{array}$ & $\begin{array}{c}\text { Altern. } \\
\mathbf{1}\end{array}$ & $\begin{array}{c}\text { Altern. } \\
\mathbf{2}\end{array}$ & $\begin{array}{c}\text { Altern. } \\
\mathbf{3}\end{array}$ & $\begin{array}{c}\text { Altern. } \\
\mathbf{4}\end{array}$ & $\begin{array}{c}\text { Altern. } \\
\mathbf{5}\end{array}$ \\
\hline Investment costs $[€]$ & 0,0 & 3161,3 & 14634,4 & 22743,1 & 4869,3 & 16216,0 \\
\hline Annual investment costs[ $[€]$ & 0,0 & 158,1 & 731,7 & 1137,2 & 243,5 & 810,8 \\
\hline Annual operating costs $[€]$ & 3797,2 & 3378,2 & 2110,2 & 1488,4 & 2507,6 & 1731,4 \\
\hline Total annual costs $[€]$ & 3797,2 & 3536,3 & 2841,9 & 2625,6 & 2751,1 & 2542,2 \\
\hline
\end{tabular}

\section{Economic evaluation comparing heating options}

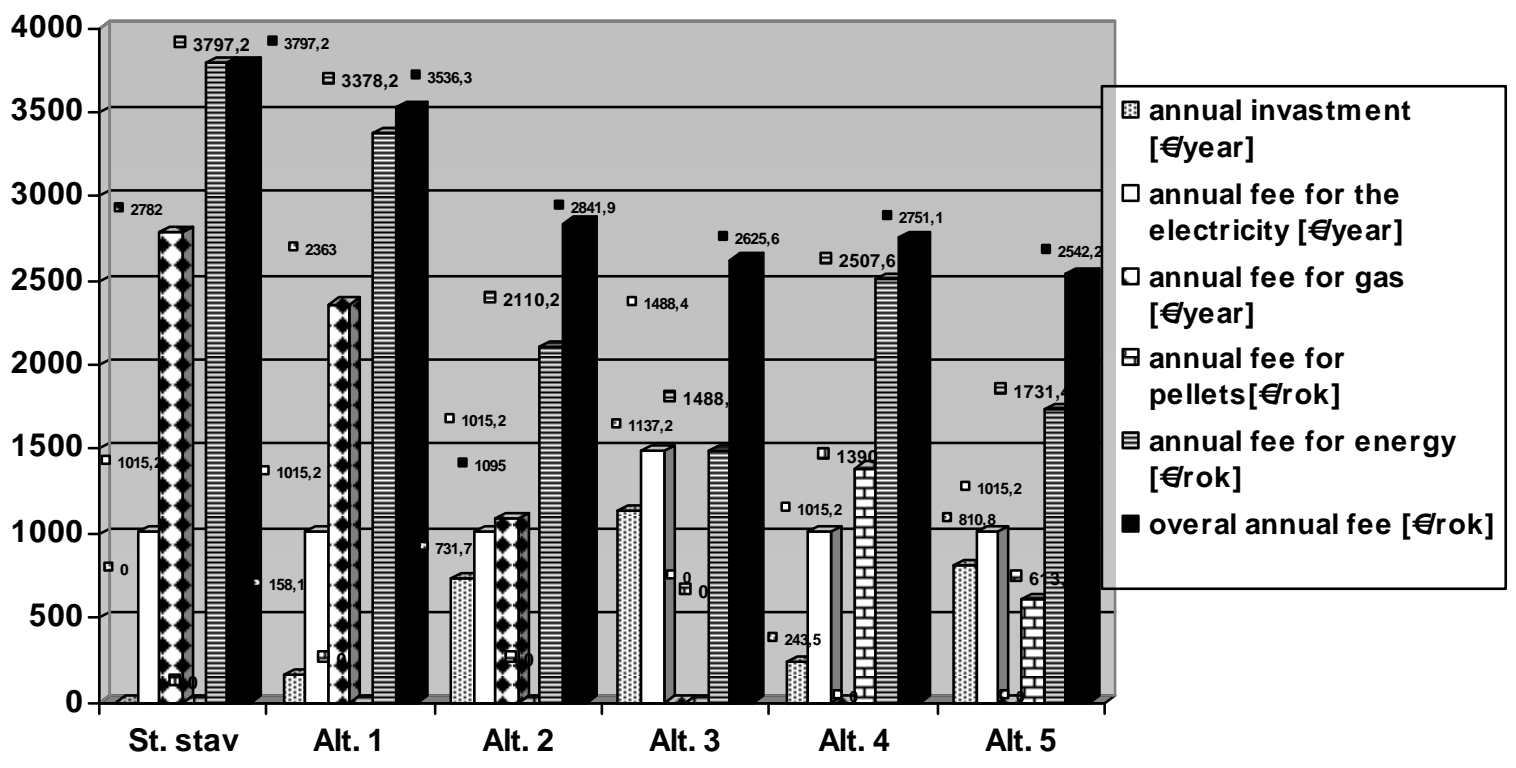

Figure 2: Evaluation of alternatives compared 
Table 6: Evaluation results comparing the various alternatives

\begin{tabular}{|c|c|c|c|c|c|c|c|c|c|c|c|c|c|c|c|c|}
\hline 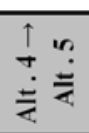 & 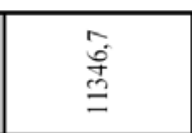 & $\begin{array}{l}1 \\
0 \\
0 \\
0 \\
= \\
=\end{array}$ & . & है & 0 & $\approx$ & iे & $\hat{n}$ & $\circ$ & 영 & & $\stackrel{\circ}{i}$ & $\begin{array}{l}0 \\
\dot{\Xi}\end{array}$ & 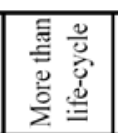 & $\begin{array}{l}0 \\
0 \\
0 \\
0 \\
0 \\
0\end{array}$ & 0 \\
\hline 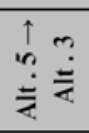 & $\vec{E}$ & 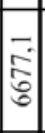 & 0 & $\hat{\mathrm{N}}$ & 0 & $\therefore$ & î & 3 & 0 & : & & के & $\begin{array}{l}\text { nे } \\
\text { ते }\end{array}$ & 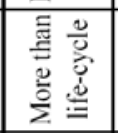 & 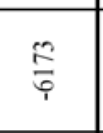 & 0 \\
\hline 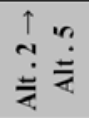 & $\begin{array}{l}\stackrel{0}{\overline{0}} \\
\stackrel{0}{\Omega}\end{array}$ & 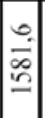 & . & $\begin{array}{l}\infty \\
\infty \\
\infty \\
\infty\end{array}$ & 0 & సิ & $\stackrel{\Xi}{\cong}$ & $\hat{n}$ & 0 & \begin{tabular}{l|} 
\\
0 \\
0 \\
0 \\
-
\end{tabular} & & $=$ & $\mathcal{F}$ & $\hat{2}$ & 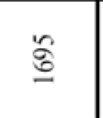 & $\underline{2}$ \\
\hline 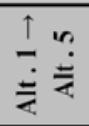 & 柋 & \begin{tabular}{l|} 
\\
$=$ \\
0 \\
0 \\
0 \\
0
\end{tabular} & 。 & 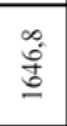 & 0 & 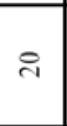 & $\stackrel{3}{=}$ & in & $\circ$ & $\begin{array}{l}0 \\
0 \\
2 \\
2\end{array}$ & 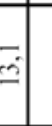 & $\overline{0}$ & $\stackrel{2}{\therefore}$ & తొ & $\stackrel{t}{\stackrel{t}{E}}$ & $\stackrel{\therefore}{\therefore}$ \\
\hline 品 & $\stackrel{\circ}{\stackrel{\mathrm{G}}{\mathrm{G}}}$ & \begin{tabular}{|l|} 
\\
\\
\end{tabular} & 0 & 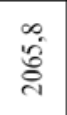 & 0 & 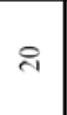 & $\Rightarrow$ & $\hat{n}^{2}$ & 0 & \begin{tabular}{|l|}
$\infty$ \\
$\frac{\infty}{二}$ \\
-
\end{tabular} & 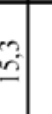 & $\stackrel{3}{\circ}$ & $\stackrel{\infty}{r}$ & ? & 亲 & $\bar{\infty}$ \\
\hline 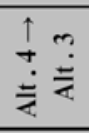 & $\begin{array}{l}\infty \\
\stackrel{\leftrightarrow}{0} \\
\stackrel{\infty}{\infty}\end{array}$ & 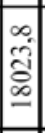 & . & $\stackrel{\check{\partial}}{\stackrel{\partial}{\circ}}$ & 0 & ชి & $\Rightarrow$ & 3 & 0 & 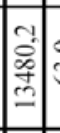 & ढे & i. & $\hat{\Xi}$ & 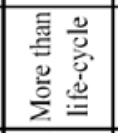 & $\stackrel{\bar{\lambda}}{\frac{\bar{n}}{7}}$ & 0 \\
\hline 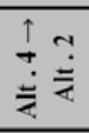 & $\begin{array}{l}\overline{0} \\
\vdots\end{array}$ & \begin{tabular}{|l|} 
\\
5 \\
2 \\
2 \\
\end{tabular} & . & 范 & 0 & ¿ิ & ¿े: & $\hat{n}^{2}$ & 0 & \begin{tabular}{|l|} 
\\
$\ddot{\infty}$ \\
$\infty$ \\
\end{tabular} & 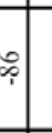 & के & $\stackrel{0}{d}$ & 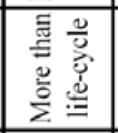 & $\begin{array}{l}\hat{\sigma} \\
\hat{0} \\
\hat{0}\end{array}$ & 0 \\
\hline 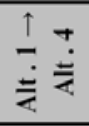 & $\stackrel{\circ}{\stackrel{\circ}{\Xi}}$ & 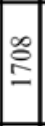 & 0 & $\stackrel{0}{\stackrel{0}{0}}$ & 0 & 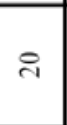 & ì & ${ }^{2}$ & 0 & $\begin{array}{l}\infty \\
\hat{\underbrace{}} \\
=\end{array}$ & : & ले & $\stackrel{\circ}{i}$ & ơ & $\frac{1}{0}$ & $\bar{r}$ \\
\hline 离导 & $\begin{array}{l}3 \\
\stackrel{3}{0} \\
\text { के }\end{array}$ & 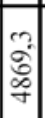 & 0 & $\begin{array}{l}0 \\
\stackrel{0}{0} \\
\stackrel{\Xi}{\Xi}\end{array}$ & 0 & तิ & $\stackrel{\mathrm{i}}{\cong}$ & 3 & 0 & 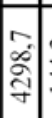 & $\begin{array}{l}y \\
\\
\end{array}$ & $\stackrel{ \pm}{=}$ & $\stackrel{\infty}{\curvearrowright}$ & $\because$ & तે̀ & $\vec{\imath}$ \\
\hline 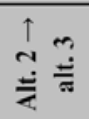 & 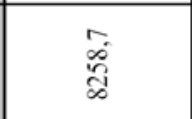 & 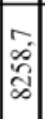 & 。 & 审 & 0 & ชิ & ì & 3 & 0 & : & $q$ & 0 & $\stackrel{\infty}{\sim}$ & $\begin{array}{l}2 \\
\infty \\
\infty\end{array}$ & ชิ & $\stackrel{0}{\circ}$ \\
\hline 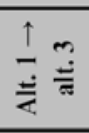 & $\frac{\infty}{\stackrel{\infty}{a}}$ & 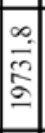 & . & $\begin{array}{l}\stackrel{\infty}{\circ} \\
\stackrel{\infty}{\infty}\end{array}$ & 0 & ๙ิ & $=3$ & $\hat{n}$ & 0 & 通 & $\div$ & ֻุ́ & $\stackrel{t}{\varrho}$ & 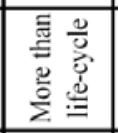 & $\begin{array}{l}\overrightarrow{0} \\
\hat{\infty} \\
\stackrel{0}{0}\end{array}$ & $\hat{m}$ \\
\hline 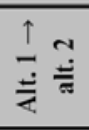 & $\begin{array}{l}\overrightarrow{\mathrm{a}} \\
\stackrel{\mathrm{I}}{=}\end{array}$ & $\begin{array}{l} \\
\text { 产 } \\
\text { = } \\
\end{array}$ & 。 & 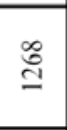 & $\circ$ & $\approx$ & जे & 3 & 0 & के & $\frac{7}{7}$ & $\bar{i}$ & $\because$ & 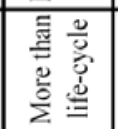 & $\begin{array}{l}\infty \\
\stackrel{\infty}{\circ} \\
\stackrel{i}{\frac{6}{7}}\end{array}$ & $\stackrel{\vec{r}}{\mathrm{r}}$ \\
\hline 离童 & $\begin{array}{l}\overrightarrow{\hat{+}} \\
\overrightarrow{\mathbf{d}}\end{array}$ & 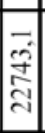 & $\circ$ & 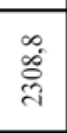 & 0 & ชิ & $=$ & $\hat{n}^{2}$ & 0 & : & ì & $\bar{\varphi}$ & $\partial$ & 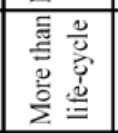 & 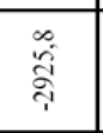 & $\stackrel{\circ}{+}$ \\
\hline 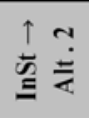 & 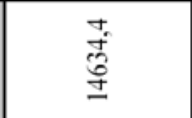 & 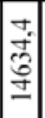 & 。 & $\ddot{\mathscr{O}}$ & 0 & 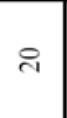 & $\Rightarrow$ & 3 & 0 & : & I & 0 & $\vec{\infty}$ & $\stackrel{2}{2}$ & $\begin{array}{l}\infty \\
\stackrel{\infty}{\varrho}\end{array}$ & $U_{0}^{+}$ \\
\hline 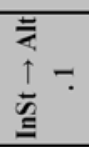 & $\frac{3}{6}$ & $\frac{3}{6}$ & 。 & $\stackrel{\rho}{\partial}$ & 0 & ิ & ते & $3^{2}$ & 0 & $\begin{array}{l} \\
\dot{\infty} \\
\frac{\alpha}{2} \\
\bar{\lambda}\end{array}$ & r. & 0 & $\because 2$ & $\stackrel{\dot{\phi}}{\underline{\partial}}$ & 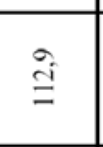 & $\approx$ \\
\hline \multirow{2}{*}{\multicolumn{3}{|c|}{ 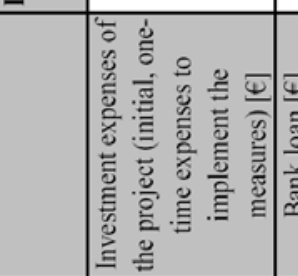 }} & \multirow{2}{*}{\multicolumn{3}{|c|}{ 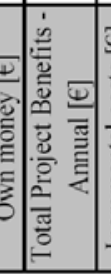 }} & \multirow{2}{*}{ 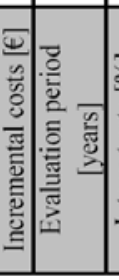 } & \multirow{2}{*}{ 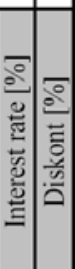 } & \multirow{2}{*}{ 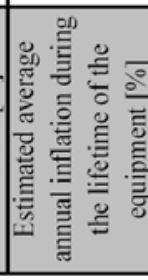 } & \multirow{2}{*}{\multicolumn{2}{|c|}{ 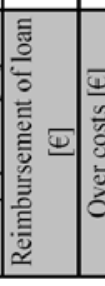 }} & & \multirow{2}{*}{ 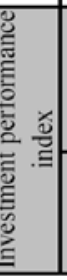 } & 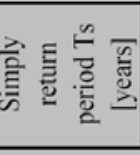 & 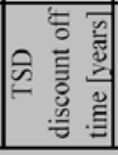 & 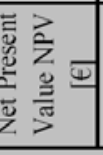 & 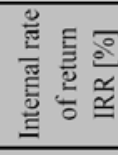 \\
\hline & & & & & & & & & & & & & \multicolumn{4}{|c|}{ 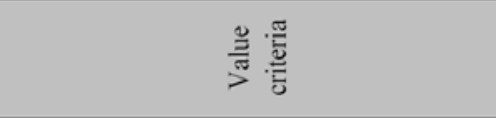 } \\
\hline
\end{tabular}


In this example, solving the heating of the house to compare returns to be combined with the old state, but this post was also for comparison between different options, in case if the house is already bearing one of the alternatives. Simple return calculation does not consider the inflation rate, respectively increase and decrease energy prices by raising the funds through a loan. This connection, however, takes into account the dynamic method.

Input parameters for the assessment of economic recovery are energy cost savings, and investments in their own actions. The calculation does not reflect the increase in energy prices. Table 6 shows the resulting values for all combinations of alternatives. Figure 3 shows the NRC process, namely net present value over the life of each combination of alternatives.

\section{Conclusion}

The alternatives under consideration is the best in terms of either simple or discounted payback period, choose alternative 4, which is clear from Figure 3. In terms of sustainability, it is appropriate to decide between alternatives 2,3 and 5 . Alternative 2 is the save fossil fuel natural gas by about two thirds compared with the old state and the sixth in comparison with the alternative 1 . In this regard, noteworthy is the choice of alternative 3 , which is used for heat pump, which shows no fossil fuel consumption, so as to lose dependence on this strategic fuel. In addition, heat pumps have the ability to heat in winter and cool in summer. It belongs to them virtually unbeatable facilities in this direction. In this paper the heat pump was "deprived" of the cooling system, which would be achieved when applying the more interesting periods of return. Upon such request would not be possible to use the existing heating system and replacing it with a device that can transfer heat and cold, would increase investment costs. Given the conservation of natural gas is best to use an alternative 5 - use of wood pellets, which we also ensure the reduction of $\mathrm{CO}_{2}$ at very attractive levels of return. 


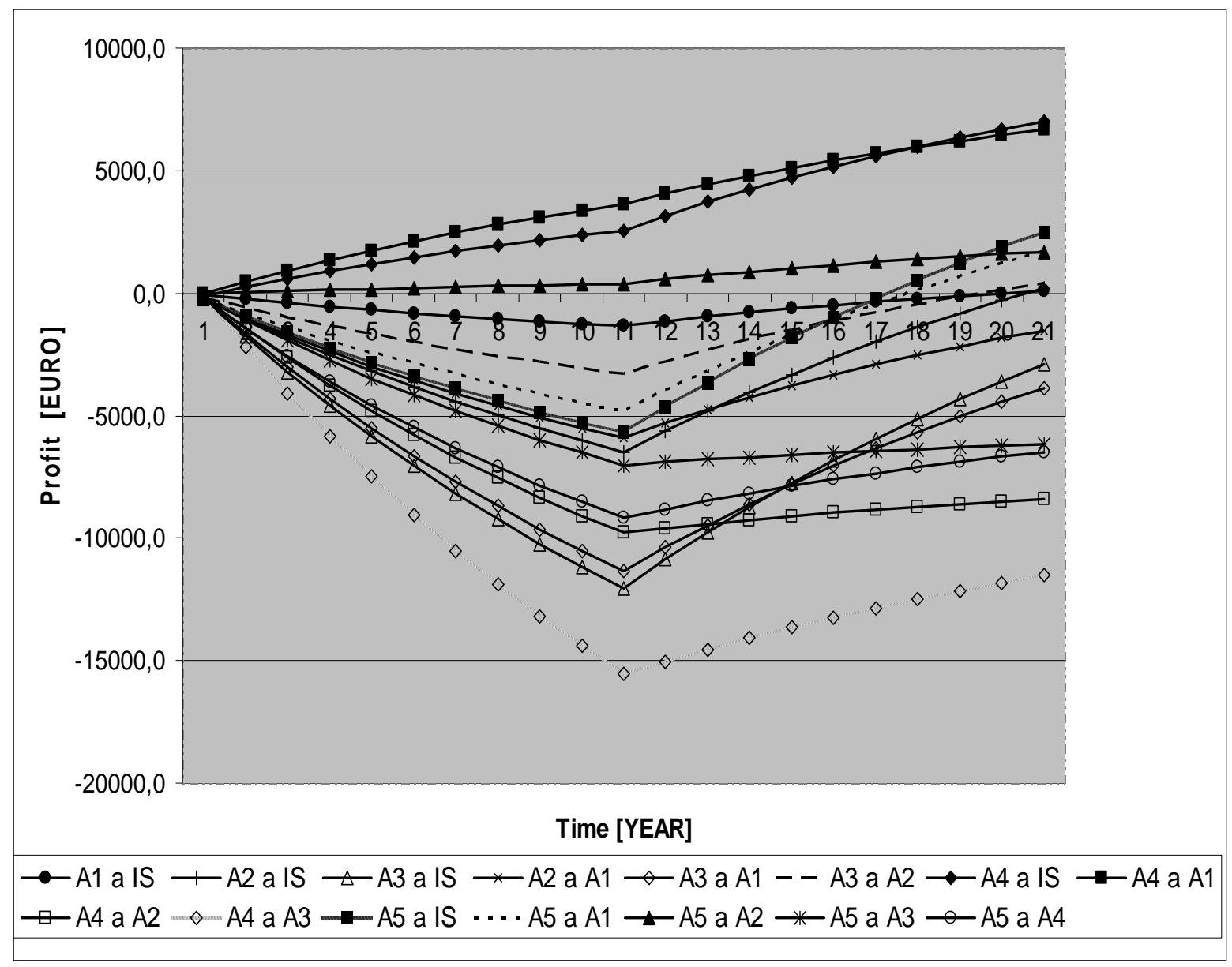

Figure 3: Net present value over the life of each combination of alternatives

\section{References}

[1] Cihelka et al. (1985). Heating, Ventilation and Air Conditioning. Praha: SNTL.

[2] STN EN ISO 13790 Thermal performance of buildings. Calculation of energy needs for heating. National Annex. Slovakia.

[3] STN EN 12831 Heating systems in buildings. The method of calculating the projected heat input. National Annex. Slovakia.

[4] STN 730540 Thermal performance of building structures and buildings. Thermal protection of buildings. Slovakia.

[5] Principle Investment. http://www.fpv.umb.sk

[6] http://new.ilava.sk/library/files/

[7] www.herz.sk, www.buderus.sk, www.rockwool.sk, www,univenta.sk, www.korad.sk, www.spp.sk, www.vse.sk 